\title{
Prestigio y estigmatización en variantes anteriorizadas y posteriorizadas de las vocales del español de Chile
}

\author{
Gastón Felipe Salamanca Gutiérrez* \\ Ana Leticia Valverde San Martín**
}

\section{Resumen}

Con esta investigación, pretendemos demostrar la pertinencia de correlacionar el parámetro fonético "dirección de la lengua" con la variable sociolingüística "estrato sociocultural". Focalizamos nuestro trabajo en el segmento etario comprendido entre los 18 y 25 años de edad, ello porque este segmento es el que exhibe una mayor consciencia de la norma lingüística y, en consecuencia, mayor distinción entre lo que se considera prestigioso y estigmatizado.

Estimamos que este trabajo constituye un aporte a los estudios sociofónicos del español (de Chile) en dos sentidos:

a. En el aspecto fonético, aporta información sobre la alofonía del inventario de vocales del español hablado en Chile y

b. En el aspecto sociolingüístico, da cuenta de la pertinencia de utilizar las nociones de prestigio y estigmatización no sólo para el inventario de consonantes -como han sido los estudios hasta aquí- sino también para el inventario de vocales.

Palabras clave: Español de Chile, alofonía vocálica, prestigio y estigmatización.

\section{Abstract}

With the following research, we intend to demonstrate the pertinence of correlating the phonetic parameter "tongue position" with the sociolinguistic variable "sociocultural stratum". We focused our work on the age group between 18 and 25 years old because that segment shows a greater awareness of the linguistics norms and, consequently, they make a clearer distinction between what is considered prestigious and stigmatized.

We believe that this work is a contribution to the sociophonetic studies of the Spanish spoken in Chile in two ways:

1. In the phonetic aspect, it gives information about the allophones in the vowel inventory of the Spanish spoken in Chile

2. In the sociolinguistic aspect, it accounts for the relevance of using the notions of status and stigmatization for not only the consonant inventory -as the researches have done so far- but the vowel inventory also.

Key words: The Spanish spoken in Chile, vowel allophones, prestige and stigmatization.

* Doctor en Lingüística, Universidad de Concepción. gsalaman@udec.cl

* Profesora de Español, Universidad de Concepción. anavalverde@udec.cl 


\section{Introducción}

Cuando los hablantes del español de Chile se refieren a la manera de hablar de una persona de estrato sociocultural alto, generalmente emplean la expresión "habla como si tuviera una papa en la boca". En el otro extremo, de una persona que pertenece a un estrato sociocultural bajo, es posible escuchar que habla "como el perrito flaite de Lipigás"1.

Estos juicios, que evidencian una valoración de los usos lingüísticos, revelan que hay algo en el habla de los sujetos que hace que sus pares los vinculen a un estrato sociocultural determinado.

En el plano de los segmentos, las investigaciones de Valdivieso (1983) y Tassara (1992), entre otras, han demostrado que hay realizaciones de ciertos fonemas consonánticos que sistemáticamente los hablantes del español de Chile asocian con el estrato sociocultural bajo. El caso más notable, sin duda, es el de la realización fricativa del fonema /t $\int /$.

Nuestra impresión es que esta valoración de los usos lingüísticos no se limita al inventario de las consonantes, sino que también afecta al inventario vocálico. Concretamente, pensamos que esta correlación se vincula con el parámetro dirección de la lengua² ${ }^{2}$ de modo que los sujetos que emplean variantes anteriorizadas son catalogados sistemáticamente por sus pares como pertenecientes a un estrato sociocultural bajo ("flaites") y aquellos que emplean variantes posteriorizadas son catalogados como pertenecientes a un estrato sociocultural alto ("cuico").

1 "Flaite", dicho grosso modo, es un término del castellano de Chile que se utiliza para referirse a una persona de estrato sociocultural (muy) bajo. Se opone a "cuico" -o, más recientemente, "peloláis"- que refiere a una persona de estrato sociocultural alto.

En el sitio de youtube <http://www.youtube.com/results?search_type=\&search_quer $\mathrm{y}=$ perro+de+lipigas\&aq=0\&oq=perro+de+li $>$ se encuentran numerosos spots publicitarios donde el perrito flaite mencionado promociona una marca de balones de gas, "hablando" en una variante subestándar del español de Chile.

2 Lo que no excluye la posibilidad de algún rasgo concomitante. 


\section{Objetivos}

\subsection{Objetivo General}

Establecer la pertinencia de correlacionar los ejes anterior/posterior del inventario de vocales del español de Chile, con las nociones de prestigio y estigmatización.

\subsection{Objetivos específicos}

a) Diseñar un instrumento que permita recoger un juicio sociolingüístico respecto de la conducta lingüística de un locutor.

b) Aplicar el instrumento a 20 sujetos, cuyas edades fluctúan entre los 18 y 25 años de edad.

c) Interpretar los datos obtenidos en términos de la pertinencia de utilizar las nociones de prestigio/estigmatización a una muestra donde se manipulan los rasgos anterior/posterior del inventario vocálico.

\section{Hipótesis}

1. Las vocales anteriorizadas se asociarán con el estrato sociocultural bajo.

2. Las vocales posteriorizadas se asociarán con el estrato sociocultural alto.

\section{Prestigio y estigmatización en el español de Chile ${ }^{3}$}

Humberto Valdivieso (1983) enfatiza que la lengua es objeto de una permanente valoración social. Así, los sujetos jerarquizan las realizaciones fónicas entre aquellas que consideran prestigiosas y aquellas que son objeto de estigmatización. También considera que es responsabilidad de la enseñanza institucionalizada de la lengua dar a conocer a los ha-

3 Dado el perfil especializado de los receptores de este trabajo, no abundamos en consideraciones fonético-fonológicas o sociolingüísticas. No obstante, señalaremos que, en el primer caso -especialmente en lo que dice relación con los criterios que se han considerado para clasificar fonéticamente una vocal-, hemos tenido a la vista los trabajos de Quilis y Fernández (1999) y Obediente (2007); en tanto que para los aspectos sociolingüísticos -especialmente, en lo que dice relación con las variables sociolingüísticas y los conceptos de prestigio y estigmatización- hemos tenido como marco de referencia los trabajos de López Morales (1993) y, sobretodo, Silva-Corvalán (2001). 
blantes cuáles son las realizaciones fónicas prestigiosas y aquellas que son valoradas negativamente por la comunidad.

Por otro lado, el mismo Valdivieso enfatiza que, dado que pertenecemos a una comunidad de hablantes, todos poseemos un conocimiento intuitivo sobre aquellos usos que son prestigiosos y aquellos que son estigmatizados. Sin embargo, estas intuiciones deben ser respaldadas con investigaciones sociolingüísticas objetivas que incorporen las variables diatópicas, diafásicas y diastráticas.

En esta misma línea, Tassara (1992) ha establecido que los usuarios nativos de una lengua tienen conciencia cultivada o ingenua de una norma lingüística y su relación con la estratificación social, lo cual implica intuir la existencia de usos estigmatizados y prestigiosos tanto en el nivel fonético de la lengua como en el léxico y morfosintáctico.

En el plano fonético, algunos de los rasgos que los investigadores reportan como caracterizadores de grupos deprivados son la asimilación de los grupos [rl] y [rn], la asibilación de [rr] y el grupo [tr], y, más que todo, la pronunciación fricativa de /t $\int /$.

Respecto de este último punto, son elocuentes dos de las conclusiones a las que arriba Tassara (1992) en su trabajo "Actitudes lingüísticas ante la variación de /ĉ/" y que reproducimos aquí:

a. La gran sensibilidad a la significación social de la fricativa [ŝ] correlacionada unánimemente con un nivel sociocultural bajo.

b. La hipersensibilidad normativa del estudiante universitario le permite, por una parte, detectar sin error al hablante inculto porque en él no sólo hay un rasgo estigmatizado sino una pluralidad de rasgos (articulación de segmentos vocálicos y consonánticos, entonación, ritmo, fluidez, dificultad de lectura, etc.). Por otra parte, estos mismos estudiantes motivados por su exacerbada conciencia normativa califican erróneamente a la pseudo-locutora $2^{4}$ por el solo hecho de presentar el rasgo estigmatizado [ŝ] fricativo. El usuario asume una actitud implacable para juzgar el habla de otro, condenando fuertemente el uso incorrecto. (Tassara, 1992: 263).

Ahora, a pesar de estos aportes, hemos advertido una carencia de trabajos que investiguen en qué medida la presencia de variantes de los fonemas vocálicos inciden en la valoración subjetiva de los hablantes. Así las cosas, basados en la intuición de que como hablantes nativos asociamos sistemáticamente ciertas realizaciones vocálicas con un estrato

4 Se hace referencia aquí a una hablante culta que practica la alternancia africada-fricativa de /ĉ/ con el predominio de la última. 
sociocultural determinado, diseñamos una investigación tendiente a confirmar o desmentir tal intuición.

\section{Metodología}

\subsection{Sujetos}

La muestra estuvo constituida por 20 sujetos, 10 de sexo masculino y 10 de sexo femenino. La edad de ellos fluctuaba entre los 18 y 24 años. Todos pertenecían a la comuna de Penco y tenían la enseñanza media concluida.

\subsection{Instrumento}

\subsubsection{Reactivos}

Se seleccionaron 5 enunciados que contenían las 5 vocales del español:
1. La pala
2. El tete
3. Mi kiwi
4. No como
5. Tu puño

Se cuidó especialmente que cada enunciado en cuestión tuviera la misma vocal, esto con la finalidad de atribuir a esa vocal un eventual cambio en la percepción de los sujetos.

Especial cuidado tuvimos también en no incluir consonantes que poseyeran realizaciones alofónicas estigmatizadas, como /t $\int / \mathrm{o} / \mathrm{rr} /$, a fin de que no fueran éstas las responsables del cambio en la percepción.

Así, entonces, dado que cada enunciado debía ser pronunciado con las vocales en tres posiciones distintas ("típica", anteriorizada y posteriorizada), la lista aumentó a 15 enunciados.

Además, decidimos incluir 15 enunciados a modo de "distractores", esto es, enunciados donde no se manipuló la variable dirección de la lengua y que, aunque poseían estructura y longitud similares a los reactivos en estudio, no necesariamente tenían las mismas vocales. Estos enunciados fueron los siguientes: 
Prestigio y estigmatización en variantes anteriorizadas y posteriorizadas de las vocales del español de Chile / Gastón Salamanca y Ana Valverde

\begin{tabular}{|c|l|l|l|l|l|}
\hline 1. & La puerta & 6. & El tigre & 11. & El mapa \\
\hline 2. & La foto & 7. & El café & 12. & El gato \\
\hline 3. & El suelo & 8. & El pato & 13. & El campo \\
\hline 4. & El libro & 9. & La copa & 14. & La silla \\
\hline 5. & La carpa & 10. & La mesa & 15. & El campo \\
\hline
\end{tabular}

De este modo, la lista final de reactivos quedó constituida por 30 enunciados, cuyo orden de aparición fue determinado al azar (ver Tabla 1).

Posteriormente, estos enunciados fueron grabados -por un locutor entrenado- en el Laboratorio de Fonética de la Universidad de Concepción.

Finamente, los reactivos se "pegaron" en el escritorio de los computadores del Laboratorio de Computación del Departamento de Español, lugar donde se aplicó el test de percepción.

Tabla No 1. Reactivos y estatus de los enunciados

\begin{tabular}{|c|c|c|}
\hline NÙMERO & ENUNCIADO & ESTATUS DEL ENUNCIADO \\
\hline 1 & La puerta & Distractor \\
\hline 2 & La foto & Distractor \\
\hline 3 & Tu puño & Con vocales posteriorizadas \\
\hline 4 & No como & Con vocales no modificadas \\
\hline 5 & La pala & Con vocales no modificadas \\
\hline 6 & El suelo & Distractor \\
\hline 7 & La pala & Con vocales anteriorizadas \\
\hline 8 & El libro & Distractor \\
\hline 9 & La carpa & Distractor \\
\hline 10 & El tete & Con vocales anteriorizadas \\
\hline 11 & El tigre & Distractor \\
\hline 12 & Tu puño & Con vocales no modificadas \\
\hline 13 & El café & Distractor \\
\hline 14 & No como & Con vocales anteriorizadas \\
\hline 15 & El pato & Distractor \\
\hline 16 & La pala & Con vocales posteriorizadas \\
\hline 17 & La copa & Distractor \\
\hline 18 & El tete & Con vocales no modificadas \\
\hline 19 & Mi kiwi & Con vocales posteriorizadas \\
\hline 20 & Tu puño & Con vocales anteriorizadas \\
\hline 21 & la mesa & Distractor \\
\hline 22 & El mapa & Distractor \\
\hline 23 & Mi kiwi & Con vocales anteriorizadas \\
\hline 24 & El tete & Con vocales posteriorizadas \\
\hline 25 & El gato & Distractor \\
\hline 26 & Mi kiwi & Con vocales no modificadas \\
\hline 27 & El campo & Distractor \\
\hline 28 & La silla & Distractor \\
\hline 29 & El punto & Distractor \\
\hline 30 & No como & Con vocales posteriorizadas \\
\hline
\end{tabular}




\subsubsection{Test de percepción}

Después de grabar los enunciados, diseñamos un test de percepción con la siguiente estructura:

\section{Instrucción:}

Usted escuchará 30 enunciados breves. Respecto de ellos, hay dos afirmaciones que usted deberá completar, cada vez, vía selección múltiple. Sólo debe seleccionar una alternativa.

Afirmación 1. Según usted, esta persona pertenece a un estrato sociocultural:
a) alto
b) bajo
c) no lo asocio con ningún estrato sociocultural en particular.

Afirmación 2. Un término de uso común que aplica para esta persona es:
a) cuico
b) flaite
c) ni flaite ni cuico, habla como
cualquier persona común y corriente.

Los sujetos debían contestar este test en una hoja de respuesta que tenía el formato que se presenta en Tabla $2^{5}$ :

Tabla No 2. Formato de hoja de respuesta

\begin{tabular}{|c|c|c|c|c|c|c|}
\hline Enunciados & \multicolumn{3}{|c|}{ Afirmación 1} & \multicolumn{3}{|c|}{ Afirmación 2} \\
\hline Enunciado 1 & A & $\mathrm{B}$ & C & A & $B$ & C \\
\hline Enunciado 2 & A & B & C & A & B & $\mathrm{C}$ \\
\hline Enunciado 3 & $\mathrm{~A}$ & B & $C$ & A & $B$ & $\mathrm{C}$ \\
\hline Enunciado 4 & A & $\mathrm{B}$ & $\mathrm{C}$ & A & $B$ & C \\
\hline Enunciado 5 & A & B & C & A & B & C \\
\hline Enunciado 6 & A & B & C & A & $B$ & C \\
\hline Enunciado 7 & A & $B$ & $C$ & A & $B$ & C \\
\hline Enunciado 8 & A & B & $C$ & A & B & $\mathrm{C}$ \\
\hline Enunciado 9 & $\mathrm{~A}$ & $B$ & $\mathrm{C}$ & A & $B$ & C \\
\hline Enunciado 10 & $A$ & $B$ & $\mathrm{C}$ & $A$ & $B$ & $\mathrm{C}$ \\
\hline
\end{tabular}

Por otra parte, nuestras expectativas respecto de la correlación dirección de la lengua-estrato sociocultural eran las que se presentan en Tabla 3.

5 Presentamos sólo los diez primeros enunciados. 
Prestigio y estigmatización en variantes anteriorizadas y posteriorizadas de las vocales del español de Chile / Gastón Salamanca y Ana Valverde

Tabla No 3. Expectativas de respuestas

\begin{tabular}{|c|c|c|}
\hline NÚMERO & ENUNCIADO & EXPECTATIVA DE RESPUESTA \\
\hline 1 & La puerta & No la asocio con ningún estrato sociocultural. C \\
\hline 2 & La foto & No la asocio con ningún estrato sociocultural. C \\
\hline 3 & Tu puño & Alto. Cuico. A. \\
\hline 4 & No como & No la asocio con ningún estrato sociocultural. C \\
\hline 5 & La pala & No la asocio con ningún estrato sociocultural. C \\
\hline 6 & El suelo & No la asocio con ningún estrato sociocultural. C \\
\hline 7 & La pala & Bajo. Flaite. B \\
\hline 8 & El libro & No la asocio con ningún estrato sociocultural. C \\
\hline 9 & La carpa & No la asocio con ningún estrato sociocultural. C \\
\hline 10 & El tete & Bajo. Flaite. B \\
\hline 11 & El tigre & No la asocio con ningún estrato sociocultural. C \\
\hline 12 & Tu puño & No la asocio con ningún estrato sociocultural. C \\
\hline 13 & El café & No la asocio con ningún estrato sociocultural. C \\
\hline 14 & No como & Bajo. Flaite. B \\
\hline 15 & El pato & No la asocio con ningún estrato sociocultural. C \\
\hline 16 & La pala & Alto. Cuico. A. \\
\hline 17 & La copa & No la asocio con ningún estrato sociocultural. C \\
\hline 18 & El tete & No la asocio con ningún estrato sociocultural. C \\
\hline 19 & Mi kiwi & Alto. Cuico. A. \\
\hline 20 & Tu puño & Bajo. Flaite. B \\
\hline 21 & La mesa & No la asocio con ningún estrato sociocultural. C \\
\hline 22 & El mapa & No la asocio con ningún estrato sociocultural. C \\
\hline 23 & Mi kiwi & Bajo. Flaite. B \\
\hline 24 & El tete & Alto. Cuico. A. \\
\hline 25 & El gato & No la asocio con ningún estrato sociocultural. C \\
\hline 26 & Mi kiwi & No la asocio con ningún estrato sociocultural. C \\
\hline 27 & El campo & No la asocio con ningún estrato sociocultural. C \\
\hline 28 & La silla & No la asocio con ningún estrato sociocultural. C \\
\hline 29 & El punto & No la asocio con ningún estrato sociocultural. C \\
\hline 30 & No como & Alto. Cuico. A. \\
\hline
\end{tabular}

\section{Resultados}

Después de la aplicación del test, se obtuvieron los resultados que se presentan en Anexos. En la parte inferior de cada casillero, aparece la alternativa seleccionada por los sujetos; en el extremo superior izquierdo, la cantidad de respuestas absolutas; y en el extremo superior derecho, el porcentaje que representa la cantidad expresada en el extremo superior izquierdo. Incluimos aquí sólo las respuesta a la afirmación 1, pues se produjo una simetría casi perfecta entre al opciones "estrato bajo/flaite", "estrato alto/cuico" y "no la asocio con ningún estrato/habla como cualquier persona común y corriente". 


\section{Interpretación de los resultados 6}

\section{Enunciado 3: "TU PUÑO" (con vocales posteriorizadas)}

- Expectativa: alternativa "A", estrato sociocultural alto.

- Tabulación de las respuestas obtenidas:

\begin{tabular}{|c|c|c|}
\hline \multicolumn{1}{|c}{ Alto } & Bajo & \multicolumn{1}{c}{ S/n asoc. con estrato } \\
\hline $420 \%$ & $525 \%$ & $1155 \%$ \\
A & B & C \\
\hline
\end{tabular}

Contrario a nuestras expectativas, sólo el $20 \%$ de los sujetos seleccionó la alternativa "A". Pensamos que esto se explica por una razón de tipo articulatoria: dado que la "u" es la vocal más posterior, no resulta posible posteriorizarla en la misma medida que las demás vocales. De ahí que los sujetos opten en su mayoría por "normal" (sin asociación a ningún estrato sociocultural), o que, advirtiendo una diferencia con respecto a la pronunciación no marcada, lo asocien en un $25 \%$ con una pronunciación de estrato sociocultural bajo.

\section{Enunciado 7: "LA PALA" (con vocales anteriorizadas)}

- Expectativa: alternativa "B", estrato sociocultural bajo.

- Tabulación de las respuestas obtenidas:

\begin{tabular}{|c|c|c|}
\hline \multicolumn{1}{|c}{ Alto } & Bajo & S/n asoc. con estrato \\
\hline $00 \%$ & $20100 \%$ & $00 \%$ \\
A & B & C \\
\hline
\end{tabular}

Este cuadro no requiere mayores comentarios. Todos y cada uno de los sujetos encuestados respondió de acuerdo a lo que habíamos previsto: que el enunciado en cuestión correspondería a un sujeto de estrato sociocultural bajo.

\section{Enunciado 10: "EL TETE" (con vocales anteriorizadas)}

- Expectativa: alternativa "B", estrato sociocultural bajo.

- Tabulación de las respuestas obtenidas:

\begin{tabular}{|c|c|c|}
\hline \multicolumn{1}{|c}{ Alto } & Bajo & \multicolumn{1}{c}{ S/n asoc. con estrato } \\
\hline $0 \%$ & $20100 \%$ & $00 \%$ \\
A & B & C \\
\hline
\end{tabular}

6 Por razones de extensión, focalizamos nuestra atención sólo en aquellos enunciados donde se modificó el parámetro dirección de la lengua. 
Al igual que para el enunciado 7, este enunciado tampoco requiere mayores comentarios, pues el $100 \%$ de los sujetos, tal como eran nuestras expectativas, lo asoció con el estrato sociocultural bajo.

\section{Enunciado 14: "NO COMO" (con vocales anteriorizadas)}

- Expectativa: alternativa "B", estrato sociocultural bajo.

- Tabulación de las respuestas obtenidas:

\begin{tabular}{|c|c|c|}
\multicolumn{1}{c}{ Alto } & Bajo & \multicolumn{1}{c|}{ S/n asoc. con estrato } \\
\hline $420 \%$ & $1050 \%$ & $630 \%$ \\
A & B & C \\
\hline
\end{tabular}

A diferencia de los enunciados 7 y 10 -donde el $100 \%$ se inclinó por la opción expectable " $\mathrm{B}$ "- en este enunciado el $50 \%$ lo hizo por esta alternativa, lo que, no obstante, constituye una preferencia mayoritaria.

En este contexto, es posible pensar en algún rasgo concomitante-por ejemplo, altura de la lengua- que también incide en la percepción de los sujetos, de modo que, en el caso de la vocal "o", lograr simultáneamente la anteriorización y el ascenso de la lengua supone un grado de dificultad que incide en su percepción 7 .

\section{Enunciado 16: "LA PALA" (con vocales posteriorizadas)}

- Expectativa: alternativa "A", estrato sociocultural alto.

- Tabulación de las respuestas obtenidas:

\begin{tabular}{|c|c|c|}
\multicolumn{1}{c}{ Alto } & Bajo & \multicolumn{1}{c|}{ S/n asoc. con estrato } \\
\hline $1470 \%$ & $315 \%$ & $315 \%$ \\
A & B & C \\
\hline
\end{tabular}

Tal como esperábamos, los sujetos seleccionaron mayoritariamente la alternativa " $A$ ". La fluctuación equitativa entre las alternativas " $B$ " $y$ " $C$ " del resto de los sujetos refleja que los individuos escucharon algo distinto, pero que no lo asociaron consistentemente con otro estrato social, lo que en este contexto confirma la consistencia de la alternativa $A$.

7 En cualquier caso, nuestra impresión es que, cualquiera sea la razón que explique el menor porcentaje de respuestas que asocian la vocal /o/ anteriorizada con el estrato sociocultural bajo, la percepción holística por parte de los sujetos en una situación de habla "real" hace que, de ocurrir una entonación flaite, un léxico flaite o la anteriorización de algunas vocales, el habla se juzgue como un todo (y no, por ejemplo, vocal por vocal); de modo que, si se presentan (algunos de) estos rasgos, se percibirá una "/o/ flaite", al mismo nivel de las incuestionablemente estigmatizadas /a/ y /e/ anteriorizadas a las que nos hemos referido en los dos casos precedentes. 


\section{Enunciado 19: "MI KIWI" (con vocales posteriorizadas)}

- Expectativa: alternativa "A", estrato sociocultural alto.

- Tabulación de las respuestas obtenidas:

Alto Bajo $\mathrm{S} / \mathrm{n}$ asoc. con estrato

\begin{tabular}{|c|c|c|}
\hline $525 \%$ & $630 \%$ & $945 \%$ \\
$\mathrm{~A}$ & $\mathrm{~B}$ & $\mathrm{C}$ \\
\hline
\end{tabular}

Distinto de lo que esperábamos, este enunciado no se vinculó con el estrato sociocultural alto. Esto se debe principalmente a que la palabra "kiwi" no fue fácilmente reconocida por los sujetos encuestados. Así, éstos intentaban prioritariamente determinar el significado del enunciado, distrayéndose del juicio respecto del estrato sociocultural ${ }^{8}$.

\section{Enunciado 20: "TU PUÑO" (con vocales anteriorizadas)}

- Expectativa: alternativa "B", estrato sociocultural bajo.

- Tabulación de las respuestas obtenidas:

\begin{tabular}{|c|c|c|}
\multicolumn{1}{|c}{ Alto } & Bajo & S/n asoc. con estrato \\
\hline $15 \%$ & $1995 \%$ & $00 \%$ \\
A & B & C \\
\hline
\end{tabular}

Tal como sucedió en 7 y 10, este enunciado no requiere mayores comentarios, puesto que el $95 \%$ de los sujetos encuestados se inclinó por la alternativa expectable " $\mathrm{B}$ ".

\section{Enunciado 23: "MI KIWI" (con vocales anteriorizadas)}

- Expectativa: alternativa "B", estrato sociocultural bajo.

- Tabulación de las respuestas obtenidas:

\begin{tabular}{|c|c|c|}
\multicolumn{1}{c}{ Alto } & Bajo & \multicolumn{1}{c}{ S/n asoc. con estrato } \\
\hline $210 \%$ & $1155 \%$ & $735 \%$ \\
A & B & C \\
\hline
\end{tabular}

Si bien mayoritariamente los encuestados seleccionaron la alternativa expectable "B", no lo hicieron en la misma medida que en los casos 7 y 10 , donde también los enunciados se vinculaban con el estrato sociocultural bajo. Pensamos que esto se explica por una razón similar a la expresada para el enunciado 3 , es decir, dado que "i" es la vocal más anterior, resulta complejo acentuar aún más este rasgo en la pronunciación.

8 En un caso concreto, uno de los sujetos nos acotó que no se distinguía bien la palabra "Manquehue". 


\section{Enunciado 24: "EL TETE" (con vocales posteriorizadas)}

- Expectativa: alternativa "A", estrato sociocultural alto.

- Tabulación de las respuestas obtenidas:

Alto Bajo $\mathrm{S} / \mathrm{n}$ asoc. con estrato

\begin{tabular}{|c|c|c|}
\hline $1680 \%$ & $15 \%$ & $315 \%$ \\
$\mathrm{~A}$ & $\mathrm{~B}$ & $\mathrm{C}$ \\
\hline
\end{tabular}

Como se observa, estos resultados no requieren mayores comentarios, pues en un porcentaje altísimo los sujetos se inclinaron por la opción prevista "A".

\section{Enunciado 30: "NO COMO" (con vocales posteriorizadas)}

- Expectativa: alternativa "A", estrato sociocultural alto.

- Tabulación de las respuestas obtenidas:

\begin{tabular}{|c|c|c|}
\multicolumn{1}{c}{ Alto } & Bajo & \multicolumn{1}{c|}{ S/n asoc. con estrato } \\
\hline $1155 \%$ & $315 \%$ & $630 \%$ \\
A & B & C \\
\hline
\end{tabular}

Si bien los encuestados seleccionaron mayoritariamente la alternativa prevista " $\mathrm{A}$ ", hay un porcentaje importante que se inclina por la alternativa " $C$ ". Explicamos este resultado por una razón similar a la expresada en el anunciado $n^{\circ} 3$, esto es, por una razón de tipo articulatoria: dado que "o" es una vocal muy posterior, no resulta sencillo posteriorizarla en la misma medida que las vocales /a/, /e/ e /i/. De ahí que los sujetos opten en un porcentaje importante por la alternativa " $\mathrm{C}$ " (sin asociación sociocultural).

\section{Conclusiones}

En primer lugar, creemos haber cumplido con los objetivos que nos planteamos. En efecto, nos parece que efectivamente es posible correlacionar los ejes anterior/posterior del inventario de vocales del español de Chile, con las nociones de prestigio/estigmatización utilizados hasta aquí para el inventario de consonantes. En términos más específicos, hemos diseñado un instrumento que permite emitir un juicio sociolingüístico respecto de la conducta lingüística de un sujeto, lo hemos aplicado al grupo previsto (20 sujetos, cuyas edades fluctuaban entre los 18 y 24 años de edad) y hemos interpretado los datos en términos de la pertinencia de utilizar las nociones de prestigio/estigmatización a una muestra donde se manipula el rasgo anterior/posterior del inventario vocálico. 
Por otro lado, nos parece que nuestras hipótesis iban en la dirección correcta, toda vez que las vocales anteriorizadas se asociaron con el estrato sociocultural bajo y las vocales posteriorizadas se asociaron con el estrato sociocultural alto. Ahora, lo interesante es que esto no se dio con un resultado de $100 \%$ en cada caso, hecho del cual se desprenden algunas consideraciones que nos parecen destacables:

1. Los dos casos en que el $100 \%$ de las respuestas fueron en una dirección, se vinculaban con el estrato sociocultural bajo. Esto confirma la idea de que nuestra población es particularmente sensible al habla de este estrato sociocultural.

2. Los casos donde se produjeron los porcentajes más altos de respuestas expectables fueron aquellos donde se modificó la dirección de la lengua en las vocales /a/ y /e/. Esto es interesante porque estas vocales son las más frecuentes en nuestra lengua, por lo que podríamos suponer que si éstas están presentes en el decurso teñirán la percepción del habla de un sujeto en su totalidad.

3. Muy vinculado con lo anterior, no debemos perder de vista que los enunciados seleccionados para esta investigación se escogieron con la finalidad de controlar lo mejor posible las variables, pero el habla "real" es mucho más compleja en su realización y holística en su percepción. Esto implica que, junto con la realización de los fonos segmentales, hay otros aspectos del habla, como la entonación y el léxico, que tributan simultáneamente para la percepción "flaite" o "cuica" del habla.

Así, por ejemplo, aunque no sea posible posteriorizar significativamene una vocal como la /u/ (o la /o/) en un enunciado aislado y, en consecuencia, no se evalúe por el $100 \%$ de los sujetos como una vocal característica de un habla "cuica" en un experimento como el nuestro, la ocurrencia en el habla "real" de las vocales "a" $y$ "e" posteriorizadas -junto con rasgos de entonación y léxico- hacen que la percepción sea de un habla "cuica" o "flaite" como una totalidad, es decir, sin distingos específicos para el caso de cada fonema vocálico.

4. La vinculación del eje anterior-posterior con la percepción "cuica" o "flaite" no descarta la incidencia de otros posibles rasgos. En efecto, pareciera ser que en el habla "cuica" la nasalización juega también un rol; en tanto que en el habla "flaite" la altura de la lengua pareciera incidir también en la percepción. Son caminos que otras investigaciones podrían explorar.

5. Sin duda, este trabajo puede ser el punto de partida para investigaciones de mayor envergadura. En ella se podrían considerar, entre otros, aspectos tales como:

a) ampliación de los rasgos fonéticos y variables sociolingüísticas considerados. 
b) aplicación de instrumentos complementarios que evalúen también la percepción holística de los sujetos.

6. Por último, no debemos olvidar que las investigaciones en lingüística pueden dejar "residuos" (zonas no resueltas cabalmente), pues estamos en presencia de un fenómeno de "baja definición". Lo importante es hacer de estos residuos nuevos focos de atención para futuras investigaciones.

\section{Bibliografía}

Figueroa, M. (2008).

Garvin, P. (1979).

López, H. (1993).

Navarro, T. (1999 [1961]).

Obediente, E. (2007).
Prestigio de las variantes de /tr/ en la comuna de Concepción. Estudio sociolingüístico. Seminario de Título para optar al grado de Licenciado en Educación con Mención en Español. Universidad de Concepción.

"Una epistemología empirista para la lingüística", en Revista de Lingüística Teórica y aplicada N. ${ }^{\circ} 17$, pp.109-127.

Sociolingüistica. Madrid: Gredos.

Manual de pronunciación Española. Madrid: Consejo Superior de Investigaciones Científicas.

Fonética y Fonología. Mérida: Consejo de Publicaciones de la Universidad de Los Andes.

Quilis, A. y Fernández, J. (1999). Curso de fonética y fonología españolas: para estudiantes angloamericanos. Madrid: Consejo Superior de Investigaciones Científicas.

Rivano, E. y Salamanca, G. (1997). Capítulos Introductorios en Lingüística General. Universidad de Concepción.

Silva-Corvalán, C. (1989). Sociolingüística. Teoría y análisis, Alhambra: Madrid.

(2001).

Tassara, G. (1992).

Valdivieso, H. (1983).
Sociolingüísticay Pragmática delespañol.Washington D.C.: Georgetown University Press.

"Actitudes lingüísticas ante la variación de /ĉ/", en Revista de Lingüística Teórica y aplicada N. ${ }^{\circ} 30, \mathrm{pp}$. 263-271.

“Prestigio y estigmatización: factor determinante en la enseñanza institucionalizada de la lengua materna", en Revista de Lingüística Teórica y aplicada N. ${ }^{\circ} 21$, pp. 137-142. 


\section{Anexos}

\section{Tabulación de los resultados}

\begin{tabular}{|c|c|c|c|}
\hline ENUNCIADOS & \multicolumn{3}{|c|}{ AFIRMACIÓN 1} \\
\hline ENUNCIADO 1 & $\begin{array}{c}630 \% \\
\mathrm{~A}\end{array}$ & $\begin{array}{c}00 \% \\
\text { B }\end{array}$ & $\begin{array}{c}1470 \% \\
C\end{array}$ \\
\hline ENUNCIADO 2 & $\begin{array}{c}420 \% \\
\mathrm{~A}\end{array}$ & $\begin{array}{c}15 \% \\
B\end{array}$ & $\begin{array}{c}1575 \% \\
\text { C }\end{array}$ \\
\hline ENUNCIADO 3 & $\begin{array}{c}420 \% \\
A\end{array}$ & $\begin{array}{c}525 \% \\
B\end{array}$ & $\begin{array}{c}1155 \% \\
C\end{array}$ \\
\hline ENUNCIADO 4 & $\begin{array}{c}630 \% \\
A\end{array}$ & $\begin{array}{c}315 \% \\
\text { B }\end{array}$ & $\begin{array}{c}1155 \% \\
\text { C }\end{array}$ \\
\hline ENUNCIADO 5 & $\begin{array}{c}210 \% \\
\mathrm{~A}\end{array}$ & $\begin{array}{c}420 \% \\
\text { B }\end{array}$ & $\begin{array}{c}1470 \% \\
\text { C }\end{array}$ \\
\hline ENUNCIADO 6 & $\begin{array}{c}630 \% \\
\mathrm{~A}\end{array}$ & $\begin{array}{c}15 \% \\
B \\
\end{array}$ & $\begin{array}{c}1365 \% \\
\mathrm{C}\end{array}$ \\
\hline ENUNCIADO 7 & $\begin{array}{c}00 \% \\
\mathrm{~A}\end{array}$ & $\begin{array}{c}20100 \% \\
\text { B }\end{array}$ & $\begin{array}{c}00 \% \\
\mathrm{C}\end{array}$ \\
\hline ENUNCIADO 8 & $\begin{array}{c}945 \% \\
\mathrm{~A}\end{array}$ & $\begin{array}{c}15 \% \\
B \\
\end{array}$ & $\begin{array}{c}1050 \% \\
\mathrm{C}\end{array}$ \\
\hline ENUNCIADO 9 & $\begin{array}{c}945 \% \\
\mathrm{~A}\end{array}$ & $\begin{array}{c}00 \% \\
\mathrm{~B}\end{array}$ & $\begin{array}{c}1155 \% \\
\mathrm{C}\end{array}$ \\
\hline ENUNCIADO 10 & $\begin{array}{c}00 \% \\
\mathrm{~A}\end{array}$ & $\begin{array}{c}20100 \% \\
\text { B }\end{array}$ & $\begin{array}{c}00 \% \\
\mathrm{C}\end{array}$ \\
\hline ENUNCIADO 11 & $\begin{array}{c}1155 \% \\
\mathrm{~A}\end{array}$ & $\begin{array}{c}00 \% \\
B\end{array}$ & $\begin{array}{c}945 \% \\
\mathrm{C}\end{array}$ \\
\hline ENUNCIADO 12 & $\begin{array}{c}210 \% \\
\mathrm{~A}\end{array}$ & $\begin{array}{c}210 \% \\
\text { B }\end{array}$ & $\begin{array}{c}1680 \% \\
\text { C }\end{array}$ \\
\hline ENUNCIADO 13 & $\begin{array}{c}1050 \% \\
\mathrm{~A}\end{array}$ & $\begin{array}{c}15 \% \\
B \\
\end{array}$ & $\begin{array}{c}945 \% \\
\mathrm{C}\end{array}$ \\
\hline ENUNCIADO 14 & $\begin{array}{c}420 \% \\
\mathrm{~A} \\
\end{array}$ & $\begin{array}{c}1050 \% \\
\text { B }\end{array}$ & $\begin{array}{c}630 \% \\
C\end{array}$ \\
\hline ENUNCIADO 15 & $\begin{array}{c}420 \% \\
\text { A }\end{array}$ & $\begin{array}{c}210 \% \\
\text { B }\end{array}$ & $\begin{array}{c}1470 \% \\
\text { C }\end{array}$ \\
\hline ENUNCIADO 16 & $\begin{array}{c}1470 \% \\
\mathrm{~A} \\
\end{array}$ & $\begin{array}{c}315 \% \\
\text { B } \\
\end{array}$ & $\begin{array}{c}315 \% \\
\mathrm{C} \\
\end{array}$ \\
\hline ENUNCIADO 17 & $\begin{array}{c}315 \% \\
\mathrm{~A}\end{array}$ & $\begin{array}{c}315 \% \\
\text { B }\end{array}$ & $\begin{array}{c}1470 \% \\
\text { C }\end{array}$ \\
\hline ENUNCIADO 18 & $\begin{array}{c}315 \% \\
\mathrm{~A}\end{array}$ & $\begin{array}{c}315 \% \\
\text { B }\end{array}$ & $\begin{array}{c}1470 \% \\
\text { C }\end{array}$ \\
\hline ENUNCIADO 19 & $\begin{array}{c}525 \% \\
\mathrm{~A}\end{array}$ & $\begin{array}{c}630 \% \\
\text { B }\end{array}$ & $\begin{array}{c}945 \% \\
\mathrm{C}\end{array}$ \\
\hline ENUNCIADO 20 & $\begin{array}{c}15 \% \\
\mathrm{~A}\end{array}$ & $\begin{array}{c}1995 \% \\
\text { B }\end{array}$ & $00 \%$ \\
\hline
\end{tabular}


Prestigio y estigmatización en variantes anteriorizadas y posteriorizadas de las vocales del español de Chile / Gastón Salamanca y Ana Valverde

\begin{tabular}{|l|c|c|c|}
\hline ENUNCIADO 21 & $630 \%$ & $210 \%$ & $1260 \%$ \\
& $\mathrm{~A}$ & $\mathrm{~B}$ & $\mathrm{C}$ \\
\hline \multirow{2}{*}{ ENUNCIADO 22} & $210 \%$ & $420 \%$ & $1470 \%$ \\
& $\mathrm{~A}$ & $\mathrm{~B}$ & $\mathrm{C}$ \\
\hline \multirow{2}{*}{ ENUNCIADO 23} & $210 \%$ & $1155 \%$ & $735 \%$ \\
& $\mathrm{~A}$ & $\mathrm{~B}$ & $\mathrm{C}$ \\
\hline \multirow{2}{*}{ ENUNCIADO 24} & $1680 \%$ & $15 \%$ & $315 \%$ \\
& $\mathrm{~A}$ & $\mathrm{~B}$ & $\mathrm{C}$ \\
\hline ENUNCIADO 25 & $525 \%$ & $210 \%$ & $1365 \%$ \\
& $\mathrm{~A}$ & $\mathrm{~B}$ & $\mathrm{C}$ \\
\hline \multirow{2}{*}{ ENUNCIADO 26} & $420 \%$ & $210 \%$ & $\mathrm{C}$ \\
\hline \multirow{2}{*}{ ENUNCIADO 27} & $\mathrm{~A}$ & $\mathrm{~B}$ & $1470 \%$ \\
& $525 \%$ & $15 \%$ & $\mathrm{C}$ \\
\hline \multirow{2}{*}{ ENUNCIADO 28} & $\mathrm{~A}$ & $\mathrm{~B}$ & $1260 \%$ \\
& $735 \%$ & $15 \%$ & $\mathrm{C}$ \\
\hline \multirow{2}{*}{ ENUNCIADO 29} & $\mathrm{~A}$ & $\mathrm{~B}$ & $1260 \%$ \\
& $840 \%$ & $\mathrm{~A} 0 \%$ & $\mathrm{C}$ \\
\hline \multirow{2}{*}{ ENUNCIADO 30} & $1155 \%$ & $\mathrm{~B}$ & $630 \%$ \\
& $\mathrm{~A}$ & $\mathrm{~B}$ & $\mathrm{C}$ \\
\hline
\end{tabular}

